\title{
Numerical and experimental investigations of three-dimensional container filling with Newtonian viscous fluids
}

\author{
M.F. Tomé ${ }^{\mathrm{a}, *}$, A. Castelo ${ }^{\mathrm{a}}$, J.M. Nóbrega ${ }^{\mathrm{b}}$, O.S. Carneiro ${ }^{\mathrm{b}}$, G.S. Paulo ${ }^{\mathrm{c}}$, F.T. Pereira ${ }^{\mathrm{a}}$ \\ a Departamento de Matemática Aplicada e Estatística, Universidade de São Paulo, Brazil \\ ${ }^{\mathrm{b}}$ Institute for Polymers and Composites/I3N, Department of Polymer Engineering, University of Minho, Campus of Azurém, $4800-058$ Guimarães, Portugal \\ ${ }^{\mathrm{c}}$ Departamento de Matemática e Computação, UNESP, Presidente Prudente, Brazil
}

\section{A R T I C L E I N F O}

\section{Article history:}

Received 4 April 2013

Received in revised form 12 September 2013

Accepted 11 November 2013

Available online 25 November 2013

\section{Keywords:}

Container filling

Jet buckling

Three-dimensional free surface flows

Experimental assessment

Finite differences

\begin{abstract}
A B S T R A C T
This work employs numerical and experimental approaches to investigate three-dimensional container filling with Newtonian viscous fluids. For this purpose, a computer code developed for simulating three-dimensional free surface flows has been used. The CFD Freeflow3D code was specifically designed to deal with unsteady three-dimensional flows possessing multiple moving free surfaces. An experimental apparatus that allows the visualization of the various phenomena that can occur during the filling of containers has been constructed and employed. Experiments on container filling were carried out by varying the fluid velocity at the injection nozzle. This paper presents a computational study on container filling with Newtonian viscous fluids and employs the experimental results to validate the software. The experimental observations were compared with the predictions from the Freeflow3D code and good agreement between the two sets of results is observed. Moreover, the code predictions showed that it is capable of capturing the most relevant phenomena observed in the experiments.
\end{abstract}

(C) 2013 Elsevier Ltd. All rights reserved.

\section{Introduction}

Industries like food, cosmetic, chemical, among others, usually employ automatic filling of containers in their production lines. In these processes, it is important to fill the containers as fast as possible to achieve high production rates and, at the same time, assure that the product characteristics are not affected negatively. The filling process is usually carried out by a moving nozzle that injects the product, in a fluid form, into a container. During the filling stage, several problems can arise like jet buckling, splashing, doming, air entrapment, among others [11]. Therefore, an understanding of the filling process can be helpful to avoid these undesirable flow regimes. Indeed, many researchers (e.g. $[2,4,6,7,11,13])$ have been working on the development of tools that are capable of simulating the filling of containers with viscous fluids. In particular, a study on the filling of two-dimensional containers was presented by Tomé et al. [13]. In their work, they considered the filling of rectangular and trapezoidal 2D containers with a Newtonian viscous fluid. This study presented numerical predictions obtained by the GENSMAC code [15], which were compared with experimental observations obtained by varying the size of the nozzle and the issuing fluid velocity at the nozzle. Good

\footnotetext{
* Corresponding author.

E-mail address: murilo@icmc.usp.br (M.F. Tomé).
}

agreement between the numerical predictions and the experimental results was reported.

In this work, we consider the application of the Freeflow3D code [2] in the simulation of a three-dimensional cubic container filling with Newtonian viscous fluids. To verify the correctness of the code predictions, an experimental system developed to visualize the instantaneous filling of containers, under controlled flow conditions, was designed and built. This device was employed to study the filling of a cubic container with a corn syrup based fluid, under different filling regimes, ranging from the occurrence of evident jet buckling to situations where splashing takes place. The Freeflow3D code was then used to simulate the filling process using the same conditions employed on the experimental runs. The filling regimes obtained and the comparison of the experimental results with the numerical predictions obtained by the Freeflow3D code are discussed.

The main contributions of this work are the experimental assessment of the Freeflow3D code and to provide numerical as well as experimental results on three-dimensional container filling. These results might be useful to researchers working on this topic, as they can use both the experimental and numerical results to benchmark the correctness of their codes.

This paper is organized as follows: in the next section a description of Freeflow3D code is presented, while Section 3 deals with the illustration of the capabilities of the Freeflow3D in simulating time-dependent three-dimensional flows, that can be found during 


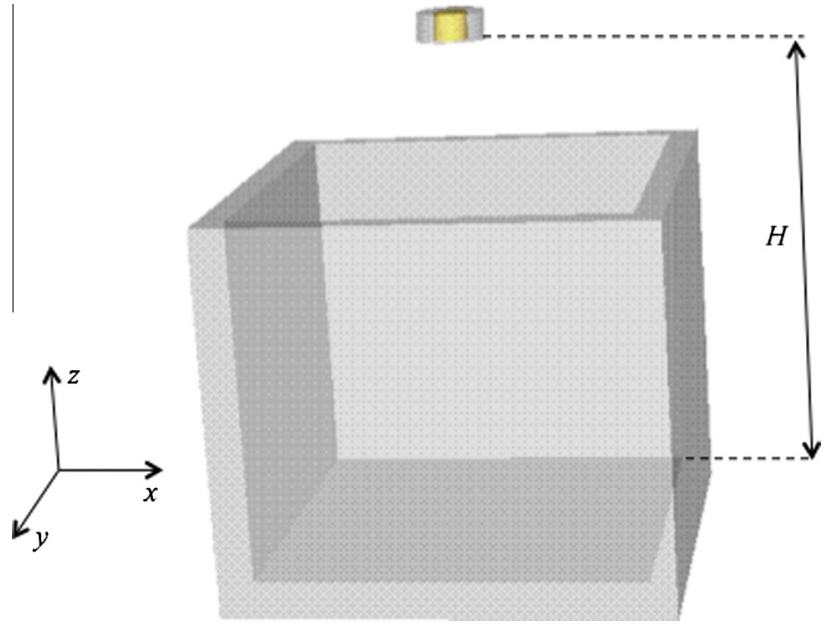

Fig. 1. Schematic view of the flow domain employed to simulate the container filling problem. The yellow surface identifies the location of the nozzle from where fluid is injected into the container. (For interpretation of the references to colour in this figure legend, the reader is referred to the web version of this article.)

the filling of a cubic container. Section 4 describes the system employed in the experiments performed on the filling of a cubic container. The experimental and the numerical results are presented, compared and analyzed in Section 5. Finally, the conclusions are summarized in Section 6.

\section{Brief description of the Freeflow3D code}

The basic equations governing incompressible unsteady moving free surface flows of Newtonian fluids are the mass conservation and the Navier-Stokes equations that can be written in nondimensional form as

$\nabla \cdot \mathbf{v}=0$

$\frac{\partial \mathbf{v}}{\partial t}=-(\mathbf{v} \cdot \nabla) \mathbf{v}-\nabla p+\frac{1}{\operatorname{Re}} \nabla^{2} \mathbf{v}+\frac{1}{\operatorname{Fr}^{2}} \mathbf{g}$,

where $t$ is the time, $\mathbf{v}$ is the fluid velocity, $p$ is the pressure, $\mathbf{g}$ is the unit gravitational field vector, $\operatorname{Re}=(\rho V D) / \mu$ is the Reynolds number and $\mathrm{Fr}=V / \sqrt{D g}$ is the Froude number. $V$ and $D$ are appropriate scalings for velocity and length, $\rho$ is the density, $\mu$ is the viscosity and $g=9.81 \mathrm{~ms}^{-2}$ is the gravitational constant.

Eqs. (1) and (2) are solved subjected to the no-slip boundary condition $(\mathbf{v}=\mathbf{0})$ on rigid walls and a prescribed velocity on inflows $\left(\boldsymbol{V}_{\text {inf }}\right)$. If surface tension is not important, the boundary conditions on the moving free-surfaces can be written as (see Batchelor [1])

$\mathbf{n} \cdot \boldsymbol{\sigma} \cdot \mathbf{n}=0$ and $\mathbf{m} \cdot \boldsymbol{\sigma} \cdot \mathbf{n}=0$,

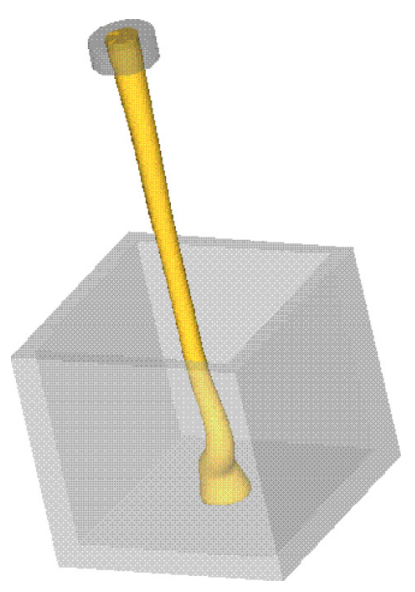

(a) $t=0.25 \mathrm{~s}$

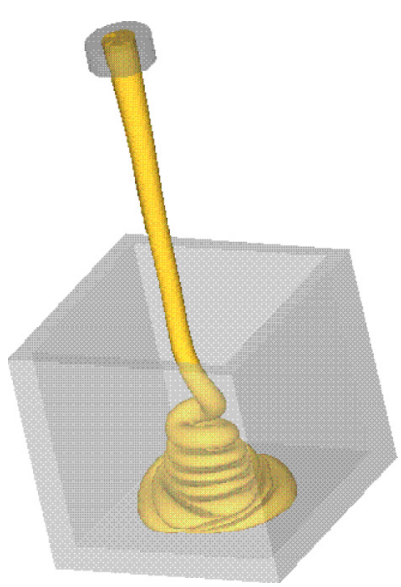

(d) $t=0.81 \mathrm{~s}$

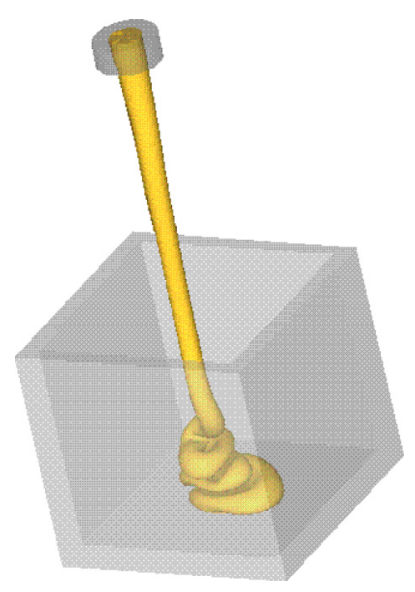

(b) $t=0.43 \mathrm{~s}$

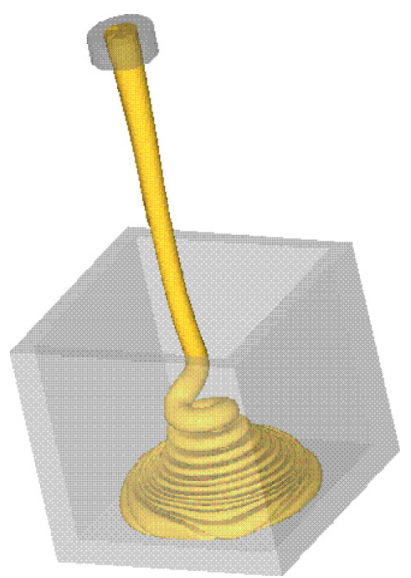

(e) $t=1.18 \mathrm{~s}$

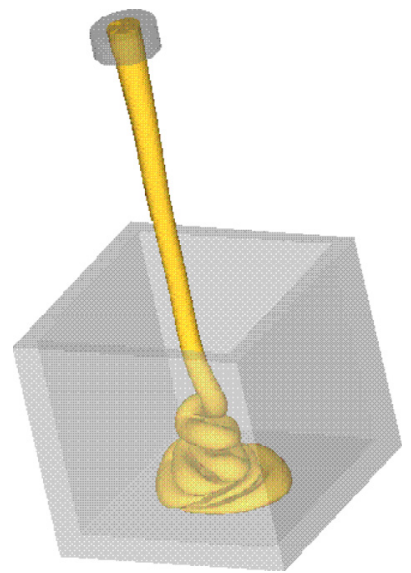

(c) $t=0.62 \mathrm{~s}$

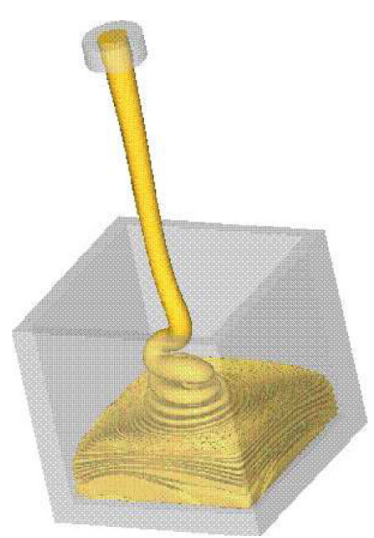

(f) $t=2.46 \mathrm{~s}$

Fig. 2. Numerical simulation of the filling of a cubic container with $V=0.25 \mathrm{~m} \mathrm{~s}^{-1}$. Fluid flow visualizations at selected times. Jet buckling is displayed. 


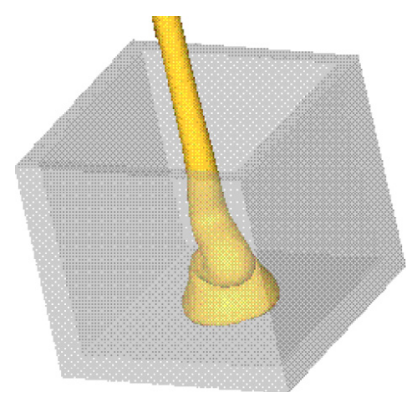

(a) $t=0.31 \mathrm{~s}$

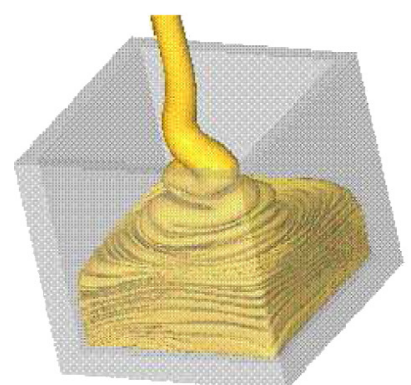

(d) $t=1.87 \mathrm{~s}$

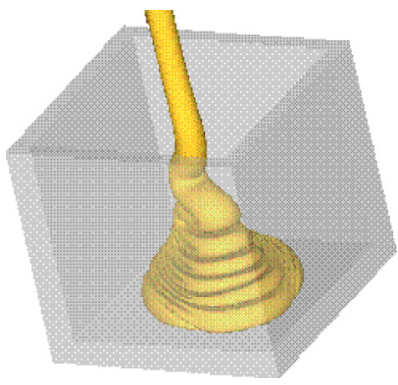

(b) $t=0.62 \mathrm{~s}$

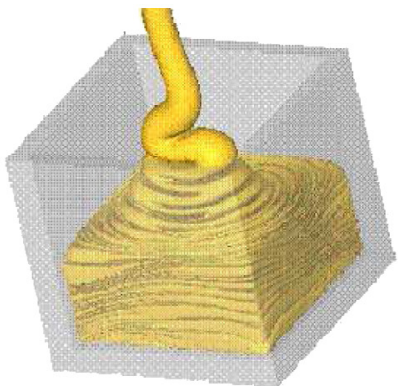

(e) $t=2.25 \mathrm{~s}$

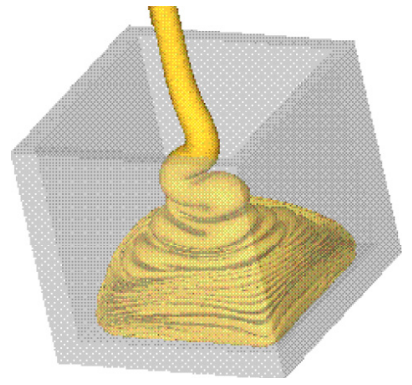

(c) $t=1.25 \mathrm{~s}$

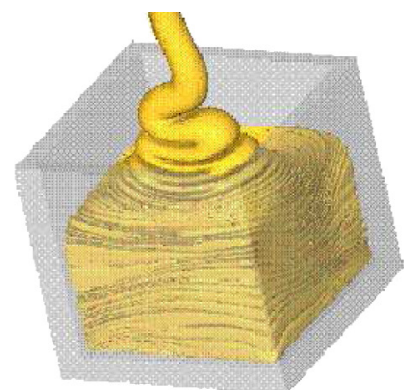

(f) $t=2.93 \mathrm{~s}$

Fig. 3. Numerical simulation of the filling of a cubic container with $V=0.5 \mathrm{~m} \mathrm{~s}^{-1}$. Fluid flow visualizations at selected times. Jet buckling is displayed.

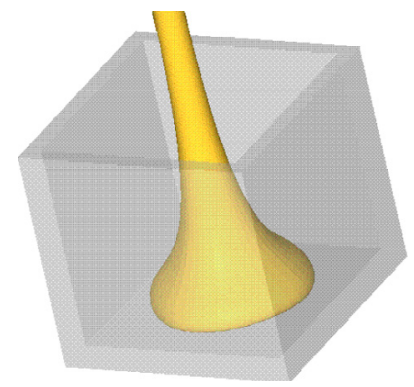

(a) $t=0.37 \mathrm{~s}$

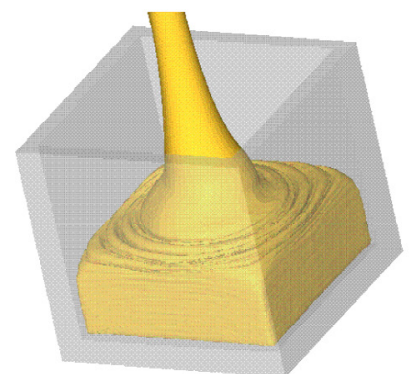

(g) $t=1.25 \mathrm{~s}$

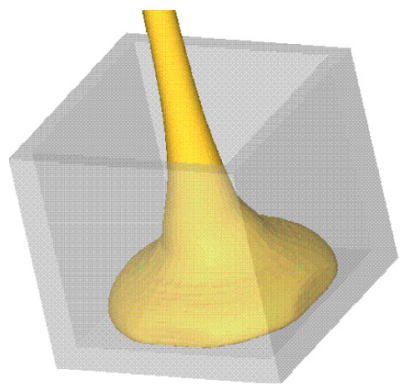

(b) $t=0.62 \mathrm{~s}$

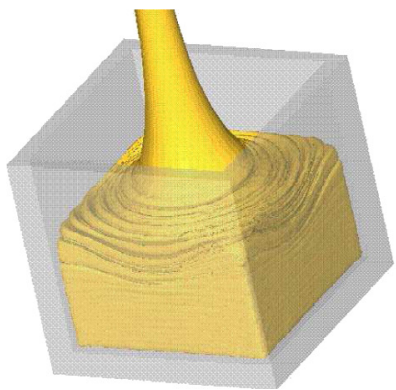

(h) $t=1.75 \mathrm{~s}$

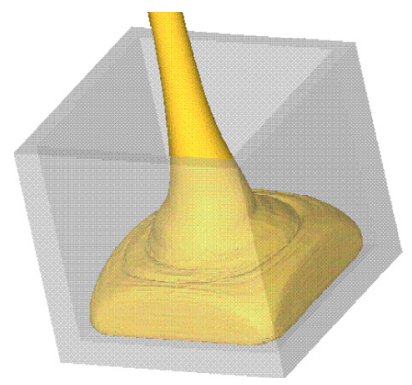

(c) $t=0.87 \mathrm{~s}$

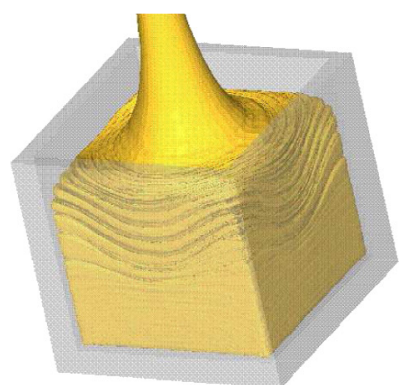

(i) $t=2.25 \mathrm{~s}$

Fig. 4. Numerical simulation of the filling of a cubic container with $V=1 \mathrm{~m} \mathrm{~s}^{-1}$. Fluid flow visualizations at selected times. Steady filling is displayed.

where $\boldsymbol{\sigma}=-p \mathbf{I}+\frac{1}{\operatorname{Re}}\left[(\nabla \mathbf{v})+(\nabla \mathbf{v})^{\mathrm{T}}\right]$ is the stress tensor and $\mathbf{n}, \mathbf{m}$ are unit vectors normal and tangential to the free surface, respectively.

Freeflow3D [2] is a code developed to simulate unsteady three-dimensional free surface flows. It embodies the GENSMAC3D method [14] and solves the Eqs. (1)-(3) using the finite difference method on a 3D-staggered grid. In GENSMAC3D, curved boundaries and free surfaces are approximated in such a way that the discrete Poisson equation, resulting from the velocity update and pressure correction, requires the solution of a symmetric positive definite linear system at each time step. This linear system, which can be large, is solved by the conjugate gradient method, in a robust and efficient manner. Details 


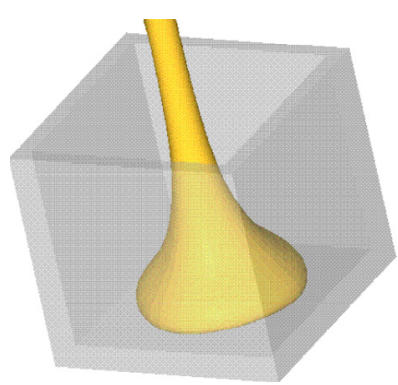

(a) $t=0.20 \mathrm{~s}$

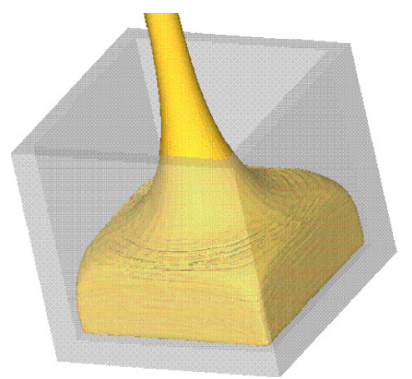

(d) $t=0.60 \mathrm{~s}$

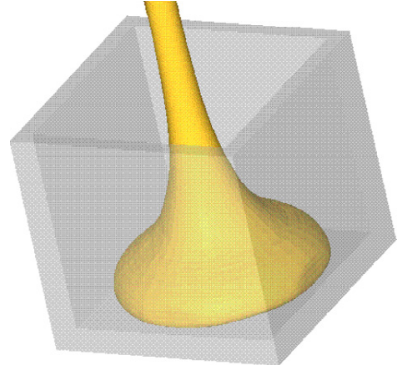

(b) $t=0.30 \mathrm{~s}$

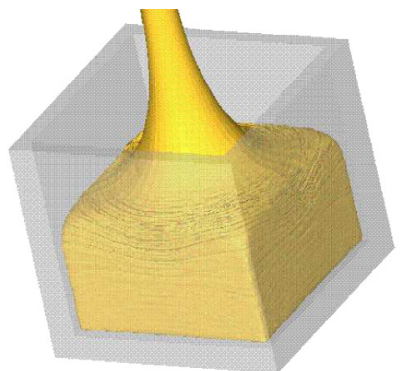

(e) $t=0.85 \mathrm{~s}$

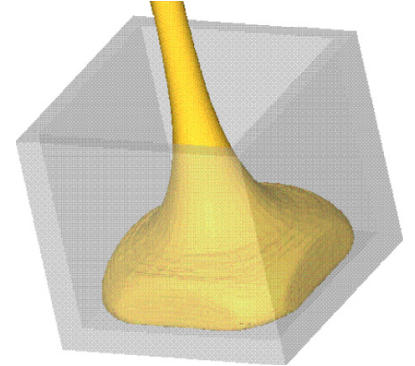

(c) $t=0.40 \mathrm{~s}$

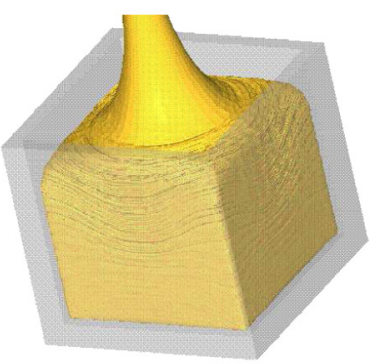

(f) $t=1.15 \mathrm{~s}$

Fig. 5. Numerical simulation of the filling of a cubic container with $V=2 \mathrm{~m} \mathrm{~s}^{-1}$. Fluid flow visualizations at selected times. Steady filling is displayed.

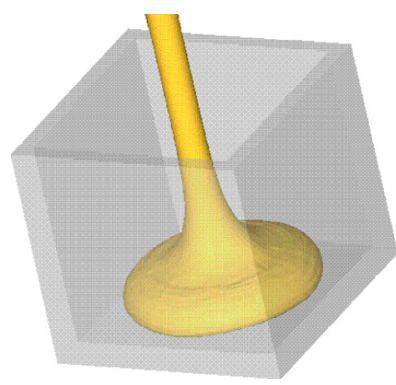

(a) $t=0.10 \mathrm{~s}$

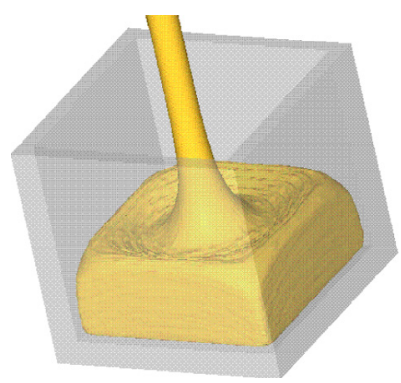

(d) $t=0.40 \mathrm{~s}$

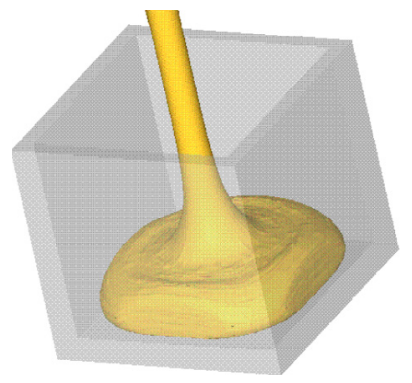

(b) $t=0.20 \mathrm{~s}$

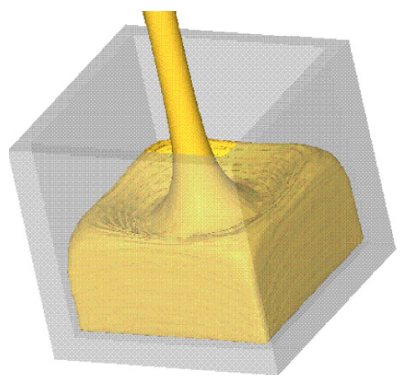

(e) $t=0.50 \mathrm{~s}$

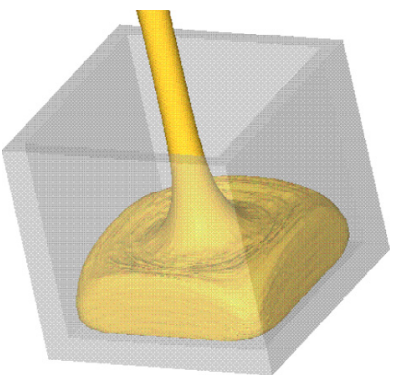

(c) $t=0.30 \mathrm{~s}$

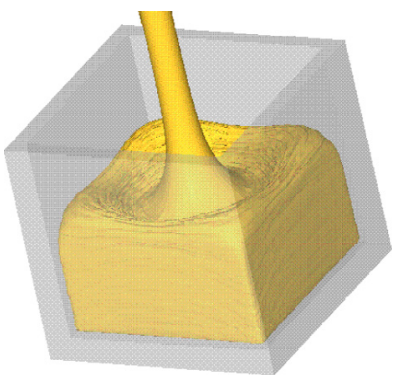

(f) $t=0.60 \mathrm{~s}$

Fig. 6. Numerical simulation of the filling of a cubic container with $V=4 \mathrm{~ms}^{-1}$. Fluid flow visualizations at selected times. Submerging regime is displayed (see [11]).

of the methodology and of the equations involved can be found in Tomé et al. [14]. In addition, the Freeflow3D code contains an interface with solid modeling technology so that a video sequence of the numerical simulations can be recorded. The Freeflow3D code comprises three distinct modular parts:
- Modflow3D - An interactive system for the specification of fluid flow models, which includes the definition of elements in the fluid domain such as containers, nozzles and fluid properties.

- Simflow3D - This module is the central part of Freeflow3D as it implements the solution of the discrete governing equations and the boundary conditions. It uses de GENSMAC3D [14] 


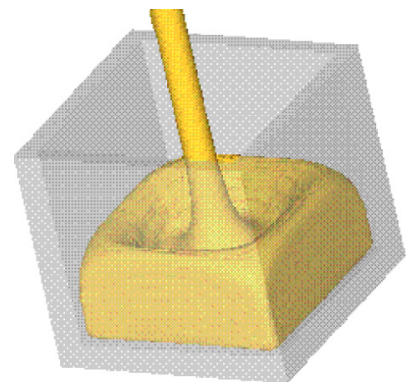

(a) $t=0.20 \mathrm{~s}$

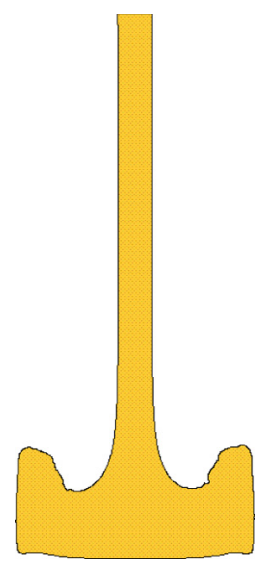

(d)

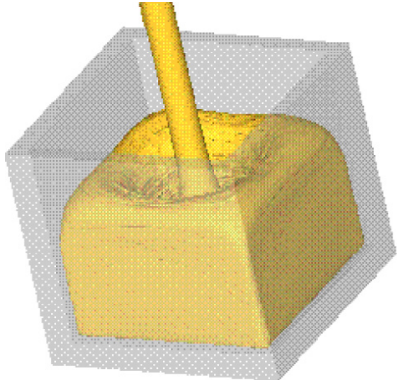

(b) $t=0.30 \mathrm{~s}$

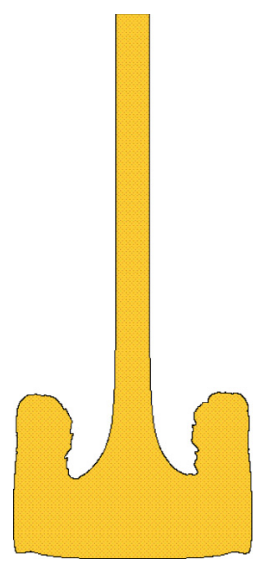

(e)

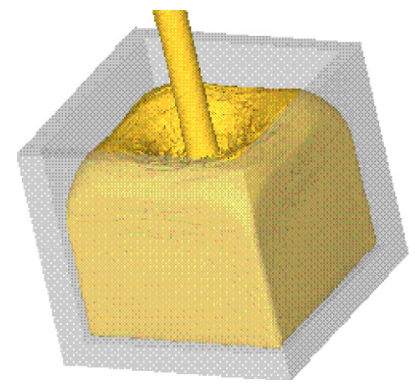

(c) $t=0.36 \mathrm{~s}$

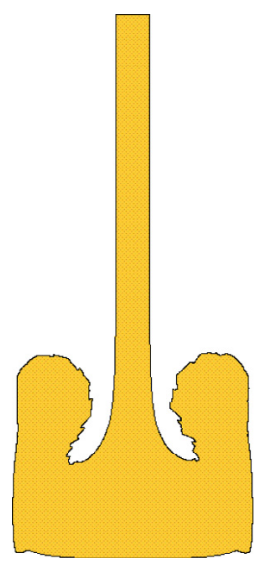

(f)

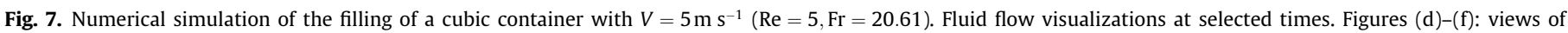
longitudinal cuts at half-width of the container at times shown in (a)-(c). Air entrapment is displayed.

method, which is a substantial enhancement of the two-dimensional GENSMAC method [15], employing marker particles only on the fluid surface.

- Visflow3D - An interactive system for the visualization of the output generated by the Simflow3D module.

A detailed description of these modules is provided in Castelo et al. [2]. The code Freeflow3D has been developed since the nineties and nowadays comprises several capabilities. For instance, it can cope with flows having high ( $\gg 1)$ [5] and low $(<1)$ [9] Reynolds numbers, flows with more than one phase [12], among others. Freeflow3D has been extensively documented $[2,9,14]$ while a review of the Marker-and-Cell method, where the capabilities of the code are discussed, has been presented by McKee et al. [8]. For this reason, details of the methodology and of the algorithm employed will not be given here.

\section{Application to 3D-container filling}

To demonstrate that the Freeflow3D code can cope with complex three-dimensional free surface flows it was applied to simulate the continuous filling of a cubic container of dimensions $(4 \times 4 \times 4) \mathrm{cm}$ with a viscous Newtonian fluid. A circular nozzle of diameter $D=0.6 \mathrm{~cm}$ positioned at a height $H=9 \mathrm{~cm}$ above the bottom of the container was employed (see Fig. 1). In the numerical experiments, the velocity $(V)$ at the nozzle was varied while the container dimensions, nozzle diameter and fluid properties were kept fixed. Gravity acted vertically in the negative $z$-direction with $g=9.81 \mathrm{~m} \mathrm{~s}^{-2}$. The following input data were employed: $\mu=6$ Pa s, $\rho=1000 \mathrm{~kg} \mathrm{~m} \mathrm{~m}^{-3}, \quad V\left(\mathrm{~m} \mathrm{~s}^{-1}\right)=0.25,1,2,3$, $4,5,10,15$. Therefore, in these simulations we had Re and Fr within the range $0.25-15$ and $1.03-61.82$, respectively, which shows that for high values of the velocity, the effect of gravity is not important. A mesh size of $(40 \times 40 \times 90)$ cells $(\delta x=\delta y=\delta z=1 \mathrm{~mm})$ was employed in all simulations. This mesh resolution was selected based on the results obtained in previous works [14] where mesh sensitivity studies were performed for similar problems.

Simulations of container filling were performed for each value of the velocity specified above. At time $t=0 \mathrm{~s}$, the fluid was injected down into the empty container at the prescribed velocity imposed at the nozzle during a specified time. An experimental analysis to predict jet buckling, provided by Cruickshank and Munson [3], establishes that an axisymmetric jet should undergo buckling if the following conditions are satisfied:

$$
\operatorname{Re}<1.2 \text { and } H / D>7.2 \text {, }
$$

where $H$ is the height of the nozzle above the bottom of the container (see Fig. 1). In these simulations, the "slenderness ratio", $H / D$, was 15 and therefore, it is expected that, when $\operatorname{Re}<1.2$ the jet is expected to become unstable, promoting the occurrence of jet buckling. Indeed, Fig. 2 displays the fluid flow configuration obtained in the simulation with $V=0.25 \mathrm{~m} \mathrm{~s}^{-1}(\mathrm{Re}=0.25, \mathrm{Fr}=1.03)$. The jet thinned due to the gravity force and soon after it impinged on the bottom of the container it became unstable (see Fig. 2a) and started buckling. At later times, after $t=0.62 \mathrm{~s}$, the jet presents the effect known as 'viscous coiling' [10] (see Figs. 2d-f). The simulation with $V=0.5 \mathrm{~m} \mathrm{~s}^{-1}(\operatorname{Re}=0.5, \mathrm{Fr}=2.06)$ also presented the buckling instability with the effect of 'jet folding' (see Fig. 3). These types of flow regimes have also been observed experimentally (see for instance Cruickshank and Munson [3] and Ribe [10]). By contrast, although the simulation with $V=1 \mathrm{~m} \mathrm{~s}^{-1} \quad(\mathrm{Re}=1, \mathrm{Fr}=4.88)$ obeyed the conditions given by Eq. (4), we can see in Fig. 4 that 


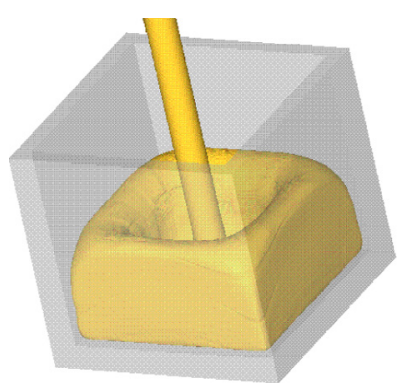

(a) $t=0.10 \mathrm{~s}$

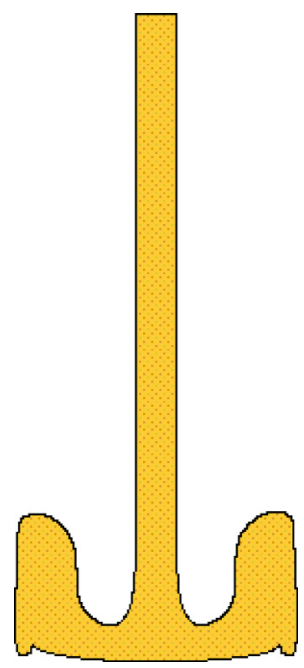

(d)

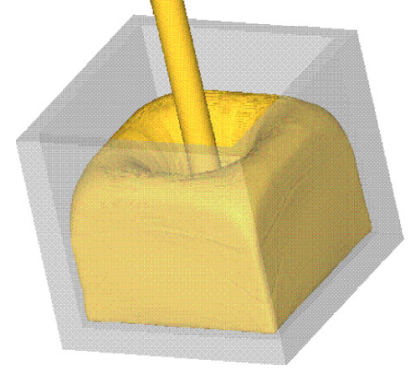

(b) $t=0.15 \mathrm{~s}$

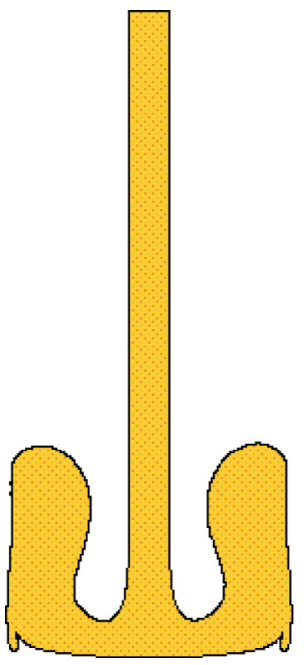

(e)

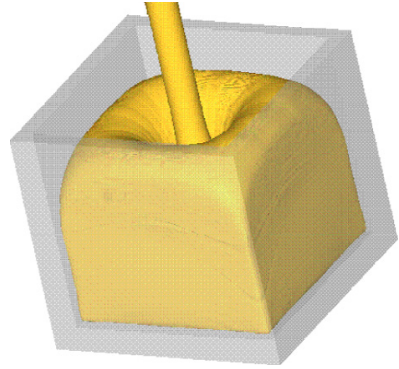

(c) $t=0.18 \mathrm{~s}$

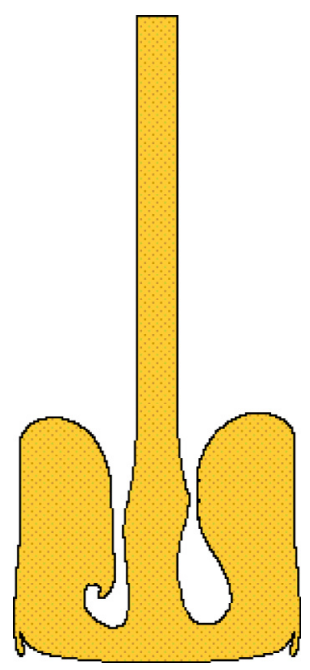

(f)

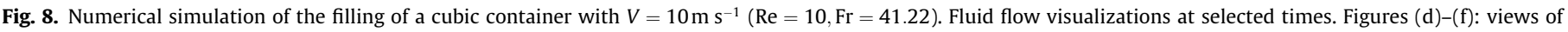
longitudinal cuts at half-width of the container at times shown in (a)-(c). Air entrapment is displayed.

the results did not display the buckling instability. Moreover, after time $t=0.62 \mathrm{~s}$ the jet developed some oscillatory motions that resulted in jet asymmetry; however, it remained stable and the filling of the container was uniform. The results from the simulation with velocity $V=2 \mathrm{~m} \mathrm{~s}^{-1}(\mathrm{Re}=2, \mathrm{Fr}=8.24)$ are displayed in Fig. 5. In this simulation the Reynolds number was higher than 1.2 so that one of the conditions in Eq. (4) is not satisfied and, therefore, buckling is not expected to occur. Indeed, as shown in Fig. 5, the jet impinges on the bottom of the container and flows radially towards the container walls without any sign of instability and the container is smoothly filled. The results obtained with $V=3 \mathrm{~m} \mathrm{~s}^{-1}$ ( $\operatorname{Re}=3, \mathrm{Fr}=12.37$ ) were similar to those displayed in Fig. 5 and therefore, are not shown. By increasing the fluid velocity at the nozzle, the results from the simulation with $V=4 \mathrm{~m} \mathrm{~s}^{-1}$ ( $\operatorname{Re}=4, \mathrm{Fr}=16.49)$ displayed in Fig. 6, show that, although the jet submerges into the fluid pool, air entrapment does not occur. For higher velocities, $V=5$ and $10 \mathrm{~m} \mathrm{~s}^{-1}((\operatorname{Re}=5, \mathrm{Fr}=20.61)$ and $(\operatorname{Re}=10, \mathrm{Fr}=41.22))$, the results shown in Figs. 7 and 8 indicate the occurrence of air entrapment and, consequently, internal air bubbles are created in the fluid pool. The results obtained with the velocity $V=15 \mathrm{~m} \mathrm{~s}^{-1}(\mathrm{Re}=15, \mathrm{Fr}=61.82)$ (not shown) were very similar to the results from the simulation with $V=10 \mathrm{~m} \mathrm{~s}^{-1}$ $(\operatorname{Re}=10, \mathrm{Fr}=41.22)$.

The numerical results obtained in this section displayed several flow regimes, that can take place during the filling of containers, known as Jet buckling (Figs. 2 and 3), Steady filling (Figs. 4 and 5), Submerging (Fig. 6) and Entrainment (Figs. 7 and 8). Similar flow regimes are presented by Roberts and Rao [11].

\section{Experimental assessment}

The experimental system designed and built to visualize the filling of containers, under controlled and reproducible conditions, was based on the system proposed by Tomé et al. [13]. The visualization device employed in the experiments is illustrated in Fig. 9a. It consists of the following parts:

(1) a reservoir to store the fluid;

(2) a nozzle with a constant cross section through which the fluid is injected into the container;

(3) a piston that can be displaced at a controlled velocity to force the fluid to flow through the nozzle at a controlled flow rate (or velocity);

(4) a transparent container that allows the visualization of the instantaneous filling process (see Fig. 9b).

(5) two moving gates that enable to close the bottom of the container. These gates allow starting the experiments employing two different modes: gate opened or gate closed. In the experiments, these gates were operated in the opened mode and then, when the jet achieved the desired fully developed flow, they were closed. Alternatively, if the objective of the experiment is to record the jet formation from the beginning of the flow, the gates were positioned in the closed mode.

Apart from the components described above, the overall experimental setup included a Universal Testing Machine INSTRON 4505 , used to control the piston velocity, and thus the fluid flow 
rate, plus a high-speed video camera to record the experimental runs. A photo of the apparatus coupled to the Universal Testing Machine is displayed in Fig. 9b.

In the experimental study performed, a corn syrup based fluid was used. Corn syrup is a common laboratory fluid adequate for this type of work. It behaves approximately as a Newtonian fluid, it can be easily obtained in the market and it is not expensive when compared to other fluids. The results presented in this work were obtained with a dilute solution of corn syrup (97\%) in water (3\%), which allowed achieving a suitable viscosity level. Viscosity measurements were performed using a ROSAND RH7-2 capillary rheometer equipped with a special device to characterize low viscosity fluids. The density of the fluid was also measured. The results obtained in the characterization of the fluid were: viscosity $\mu$ $=1.99 \mathrm{~Pa} \mathrm{~s}$ and density $\rho=1380 \mathrm{~kg} \mathrm{~m}^{-3}$. The geometry employed in the experiments was the following: circular nozzle with diameter $D=0.6 \mathrm{~cm}$; cubic recipient of dimensions $6 \times 6 \times 6 \mathrm{~cm}$; exit of the nozzle located at a distance $H=16 \mathrm{~cm}$ above the bottom of the container. Fig. 10 depicts schematically the geometry employed in the experiments. During the experiments several different filling conditions were tested, however, the comparisons with the numerical predictions are only presented for the runs listed in Table 1.

As shown in Table 1, a total of six experimental runs and corresponding numerical simulations have been carried out. A mesh size

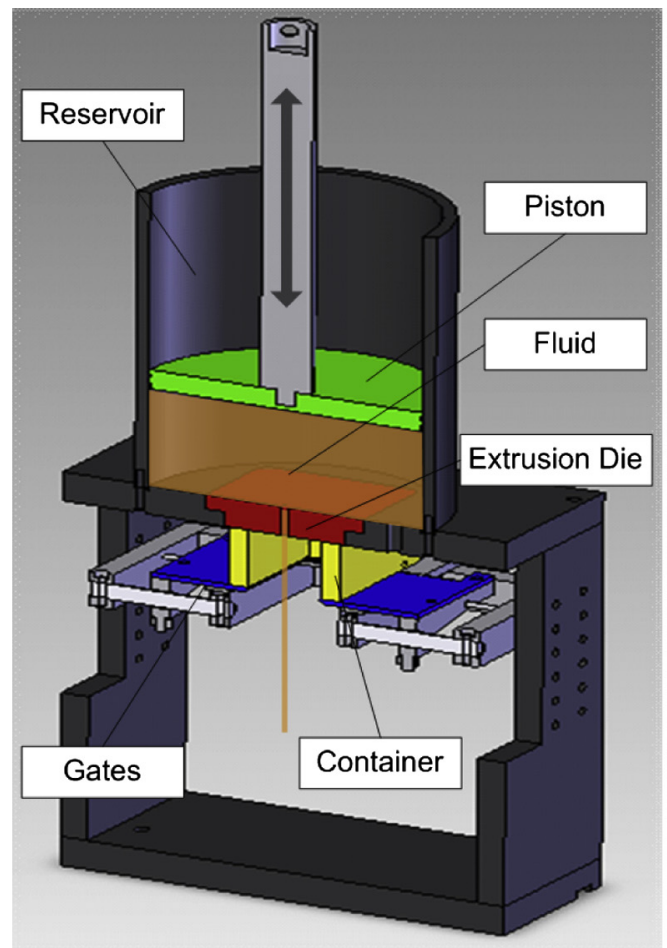

(a)

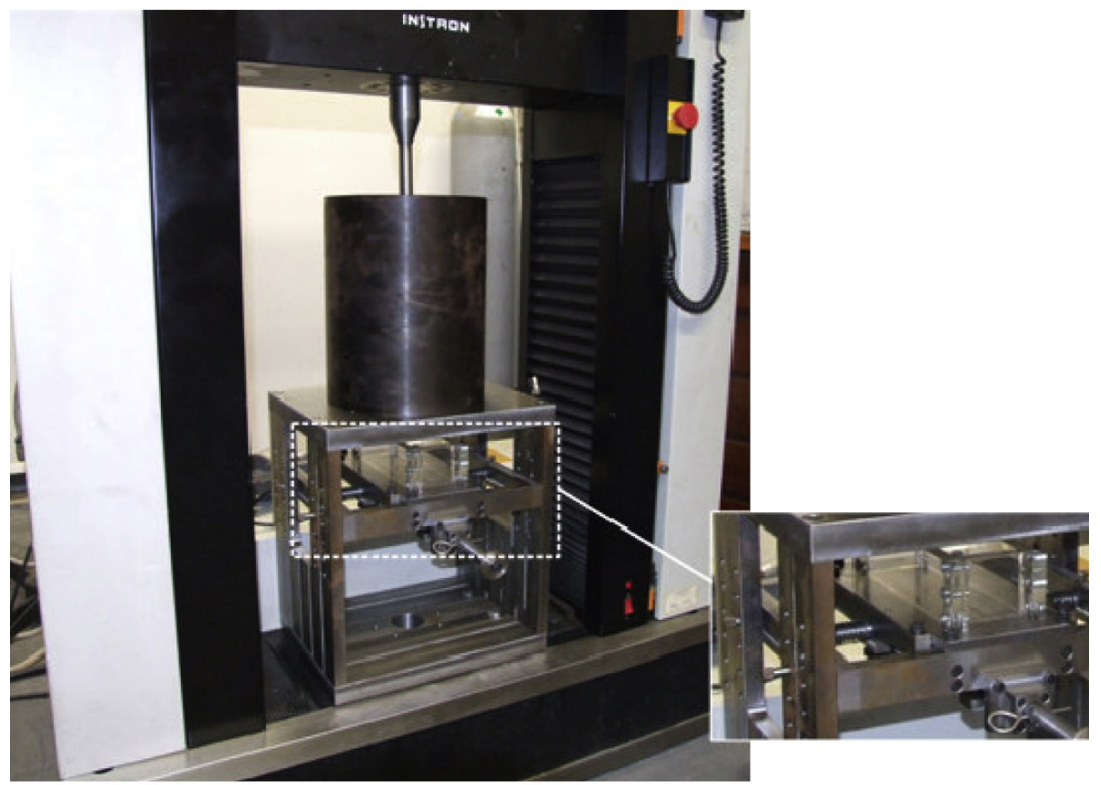

(b)

Fig. 9. Experimental apparatus: (a) Schematic view of the experimental visualization device; (b) Photo of the experimental setup. 


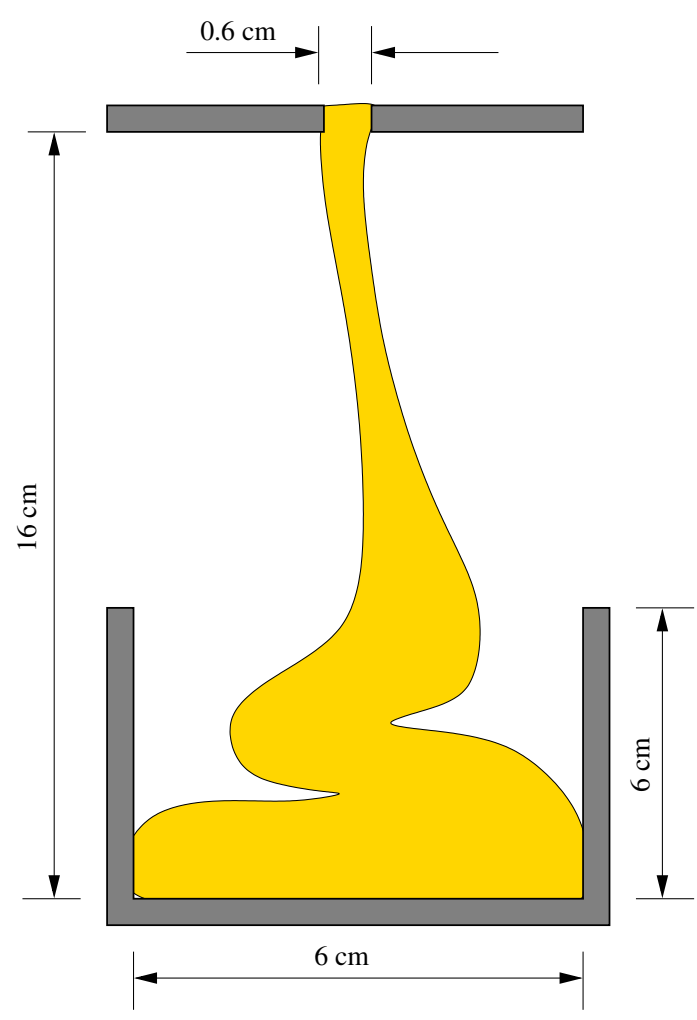

Fig. 10. Two-dimensional schematic view of the geometry employed in the experiments.

Table 1

Data employed in both numerical simulations and experimental runs on container filling.

\begin{tabular}{|c|c|c|c|c|c|}
\hline Runs & $\begin{array}{l}\text { Piston velocity } \\
\left(\mathrm{mm} \mathrm{min}{ }^{-1}\right)\end{array}$ & $\begin{array}{l}\text { Flow rate } \\
\left(\mathrm{mm}^{3} \mathrm{~s}^{-1}\right)\end{array}$ & $\begin{array}{l}\text { Nozzle velocity } \\
\left(\mathrm{m} \mathrm{s}^{-1}\right)\end{array}$ & $\begin{array}{l}R e \\
{\left[\frac{\rho \vee D}{\mu}\right]}\end{array}$ & $\begin{array}{l}F r \\
{\left[\frac{V}{\sqrt{D g}}\right]}\end{array}$ \\
\hline R1 & 5 & 3284 & 0.1161 & 0.48 & 0.48 \\
\hline R2 & 10 & 6568 & 0.2323 & 0.97 & 0.96 \\
\hline R3 & 20 & 13,136 & 0.4646 & 1.93 & 1.92 \\
\hline R4 & 50 & 32,840 & 1.1615 & 4.83 & 4.79 \\
\hline R5 & 100 & 65,680 & 2.3230 & 9.67 & 9.58 \\
\hline R6 & 250 & 164,200 & 5.8074 & 24.16 & 23.94 \\
\hline
\end{tabular}

of $(106 \times 106 \times 153)$ cells $(\delta x=\delta y=\delta z=1 \mathrm{~mm})$ was employed in each simulation. The time-step $(\delta t)$ employed in the simulations obeyed the following restrictions (see [15]):

$\begin{array}{ll}\text { (i) } \delta t<\frac{R e}{6} \delta h^{2}, & \text { (ii) } \delta t<\frac{V_{\max }}{\delta h} \text {. }\end{array}$

where $\delta h$ is the mesh spacing and $V_{\max }$ is the maximum value of the velocity field at time $t$. The simulations corresponding to the velocities $V=0.1161 \mathrm{~m} \mathrm{~s}^{-1}$ and $0.2323 \mathrm{~m} \mathrm{~s}^{-1}$, respectively runs $\mathbf{R} \mathbf{1}$ and $\mathbf{R 2}$, were forced to use a very small $\delta t$ and for this reason, it was not possible to reach a long time. The simulation with R1 stopped before the jet reached the side walls of the container while the simulation $\mathbf{R} 2$ was carried out until the jet reached the container walls. For higher velocities, the simulations were carried out until the container was half full.

\section{Results and discussion}

Some flow features observed in the experiments performed and in the corresponding numerical simulations are presented and discussed in the following sections.

\subsection{Jet thinning}

From the results obtained, one effect that can be easily observed is the "jet thinning". The Froude numbers shown in Table 1 are in the range $[0.48,23.94]$ so it is expected that, for small values of this number, the gravity force will accelerate the jet and, due to mass conservation, the jet should thin. Indeed, Fig. 11 displays the fluid flow visualization extracted, from both numerical simulations and experiments, after the jet impinged on the bottom of the container for the runs $\mathbf{R 2}, \mathbf{R 4}$ and $\mathbf{R 6}$, while, for the same runs, Fig. 12 depicts the calculated diameter of the jets at the following axial locations: $z(\mathrm{~cm})=3,9.5,16$. One can see from both Figs. 11 and 12, that for the smallest Froude number $(\mathbf{R 2})$ the jet diameter decreased substantially, while for the largest one (R6) the jet almost did not thin and maintained approximately the same diameter at the exit of the nozzle $(z=16 \mathrm{~cm})$. The diameters of the jets corresponding to runs $\mathbf{R 2}(\mathrm{Fr}=0.96), \mathbf{R} \mathbf{4}(\mathrm{Fr}=4.79)$ and R6 $(\mathrm{Fr}=23.94)$, diminished, respectively, about $42 \%, 18 \%, 1 \%$ from its initial value. These results are in excellent agreement with the experimental results displayed in Fig. 11. Moreover, the Freeflow3D results are in accordance with the model equations that predict that the smaller is the Froude number the bigger is the effect of the force of the gravity.

\subsection{Jet buckling}

It is known that, if the conditions given by Eq. (4) are satisfied, a Newtonian jet impinging on a flat plate may become unstable and the phenomenon of jet buckling should occur (see Cruickshank and Munson [3]). From Table 1 we see that R1 and R2 have Reynolds numbers of 0.48 and 0.97 , respectively, and a ratio $H / D=16 / 0.6 \approx 27$. Therefore, for these runs, it is expected that the jet may become unstable and undergo buckling. Fig. 13 displays the evolution of the fluid surface, for both the experimental and numerical trials, for run $\mathbf{R} 1(\operatorname{Re}=0.48, \mathrm{Fr}=0.48)$ at selected times. It can be seen that, soon after the jet impinged on the bottom of the container, it became unstable and started buckling. This phenomenon is observed in both the experiments and the numerical results and is in agreement with the experimental results reported by Cruickshank and Munson [3]. However, there are some dissimilarities between the experimental observations and the numerical results, that are related to the manner in which the jet buckles. In the experiment it is observed that soon after the jet buckles, the fluid accumulates onto itself, while in the numerical simulation the jet buckles and the fluid flows away from the main jet. We believe that these differences can be attributed to the fact that the fluid employed in the experiment possesses non-negligible surface tension. Fig. 14 depicts some snapshots taken from results obtained with run R2. We point out that, although the Cruickshank conditions given in Eq. (4) were fulfilled in both experimental and numerical results, jet buckling was not observed. Both results show that after impingement the jet experienced a slight instability that soon disappeared. A moving wave traveling downward the jet can also be observed in both experimental and numerical results.

\subsection{Submerging}

During the filling of a container, depending on the value of the velocity at the nozzle, the jet may partially enter the fluid pool and fill the container without the occurrence of air entrapment [11]. This flow regime, known as Submerging [11], has been simulated in Section 3. Both experimental observations and numerical results obtained for run $\mathbf{R} 3$ display an initial smooth filling process and jet submerging at later times (see Fig. 15). On the whole, a good 
R2
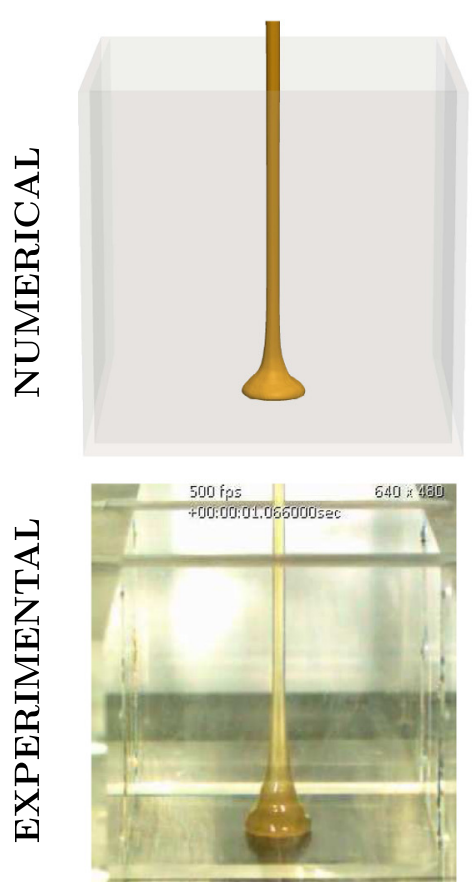

$V=0.2323 \mathrm{~ms}^{-1}$
R4
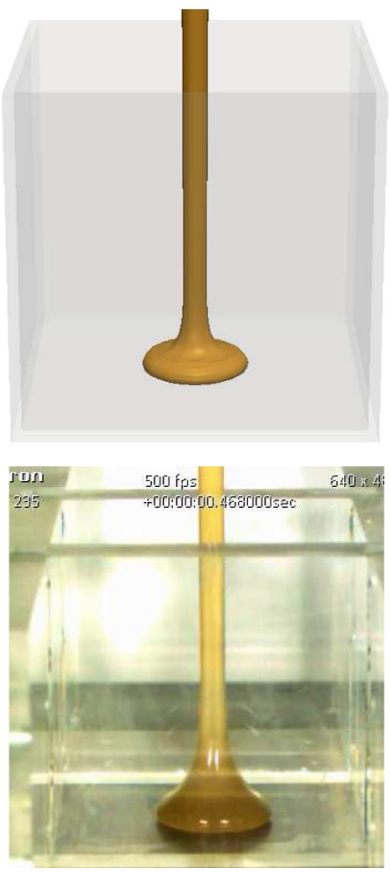

$V=1.1516 \mathrm{~ms}^{-1}$
R6
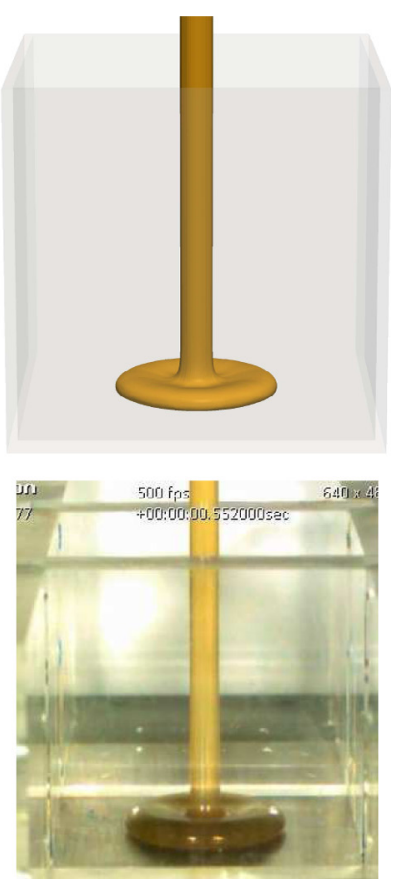

$V=5.8074 \mathrm{~ms}^{-1}$

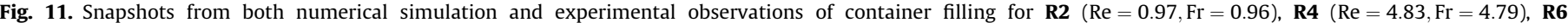
$(\operatorname{Re}=24.16, \mathrm{Fr}=23.94)$.

$\mathbf{R 2}$

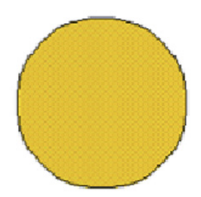

$Z=9.5 \mathrm{~cm}$

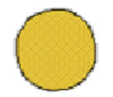

$Z=3.0 \mathrm{~cm}$
R4
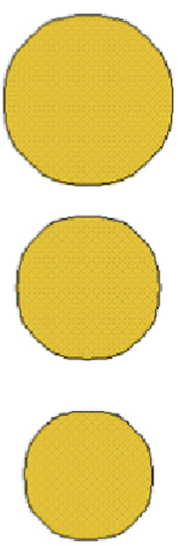

R6
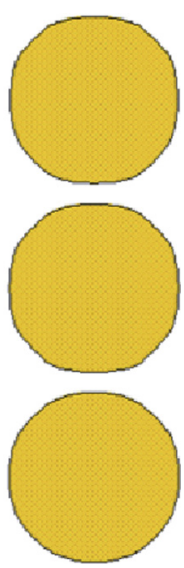

Fig. 12. Diameter of the jet measured at several heights above the bottom of the container for runs $\mathbf{R 2}(\operatorname{Re}=0.97, \mathrm{Fr}=0.96), \quad \mathbf{R 4} \quad(\operatorname{Re}=4.83, \mathrm{Fr}=4.79), \quad \mathbf{R 6}$ $(\mathrm{Re}=24.16, \mathrm{Fr}=23.94)$.

agreement between the experimental and the numerical results is again observed.

\subsection{Entrainment}

Tomé et al. [13] investigated instantaneous filling of twodimensional closed tubs with Newtonian viscous fluids. They showed that, if the velocity at the nozzle is large, the jet flows onto the bottom of the container, moves towards the side walls and splashes back causing a secondary flow that re-joins the main jet, giving rise to internal bubbles (see also [11]). This type of flow, known as Splashing, has been illustrated in the simulations presented in Section 3 (see Figs. 7 and 8).
In the simulations and experiments performed for runs $\mathbf{R 4}$ to $\mathbf{R 6}$ the results showed that air entrapment might have occurred. For example, Fig. 16 reproduces the development of the Entrainment flow regime. The agreement between the experimental results and the numerical predictions is excellent. The results obtained with velocities for runs R4 and R5 displayed similar behavior and, therefore, are not shown.

\section{Concluding remarks}

This work was concerned with numerical and experimental studies of three-dimensional container filling with Newtonian viscous fluids. A brief description of the simulation code employed, Freeflow3D, and its capabilities was provided. A study performed with the Freeflow3D code showed that it can cope with several flow regimes usually observed during filling processes, such as Jet buckling, Steady filling, Submerging and Entrainment.

With the purpose of validating the code, a new testing device to perform the filling of a cubic container was designed and built. This device was capable of promoting the filling of containers under controlled and reproducible conditions and comprises a transparent cubic container that allows recording the filling process. Numerical and experimental examinations of the filling of a cubic container through a circular nozzle were performed using a Newtonian viscous fluid based on corn syrup. The results obtained displayed various effects like jet thinning, jet buckling, jet submerging, smooth filling, splashing and entrainment. The good correlations obtained between the numerical results and experimental observations allowed to validade the Freeflow3D code. These results demonstrate that the Freeflow3D code can predict almost all phenomena observed experimentally. However, for small values of the nozzle velocity some discrepancies were observed, which were attributed to the fact that the Freeflow3D code does not incorporate surface tension on the free surface boundary condition. 

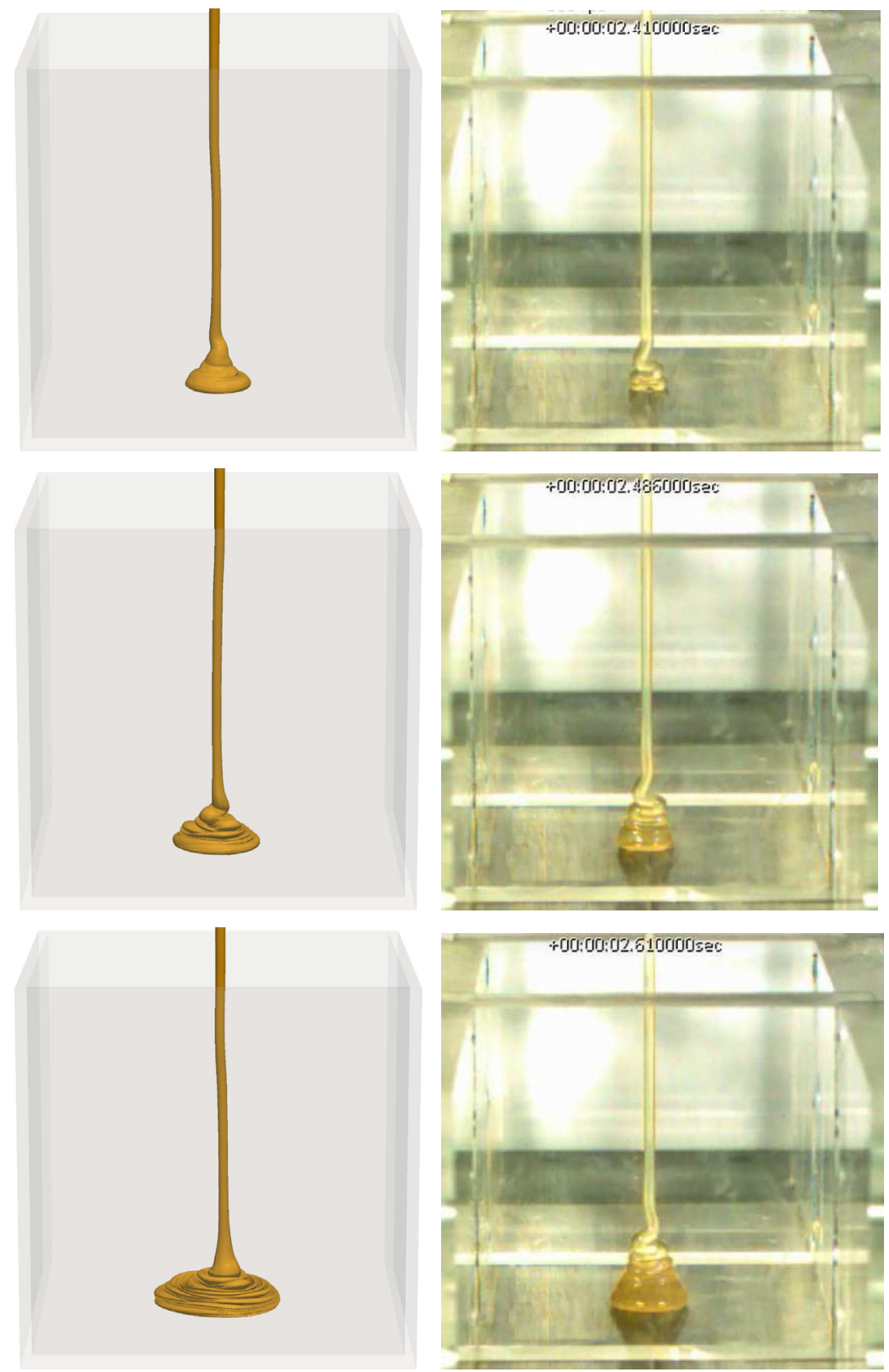

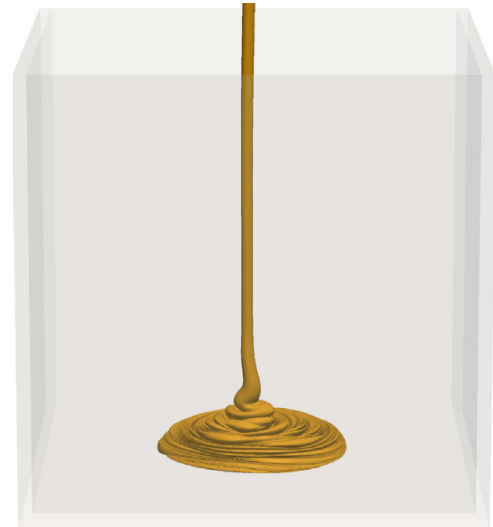

NUMERICAL

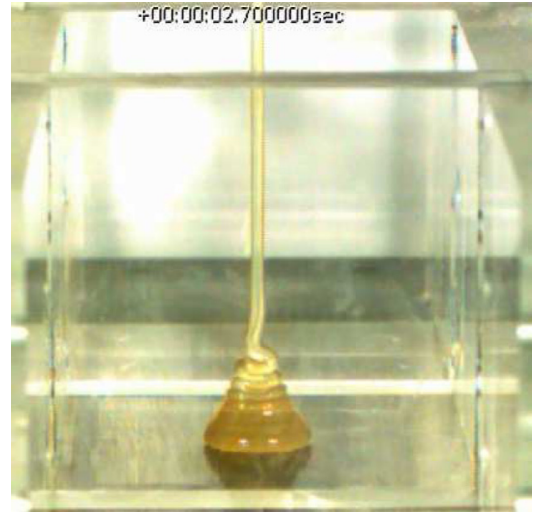

EXPERIMENTAL

Fig. 13. Snapshots from both numerical simulation and experimental observations of container filling for run $\mathbf{R 1}(\mathrm{Re}=0.48, \mathrm{Fr}=0.48$ ). 

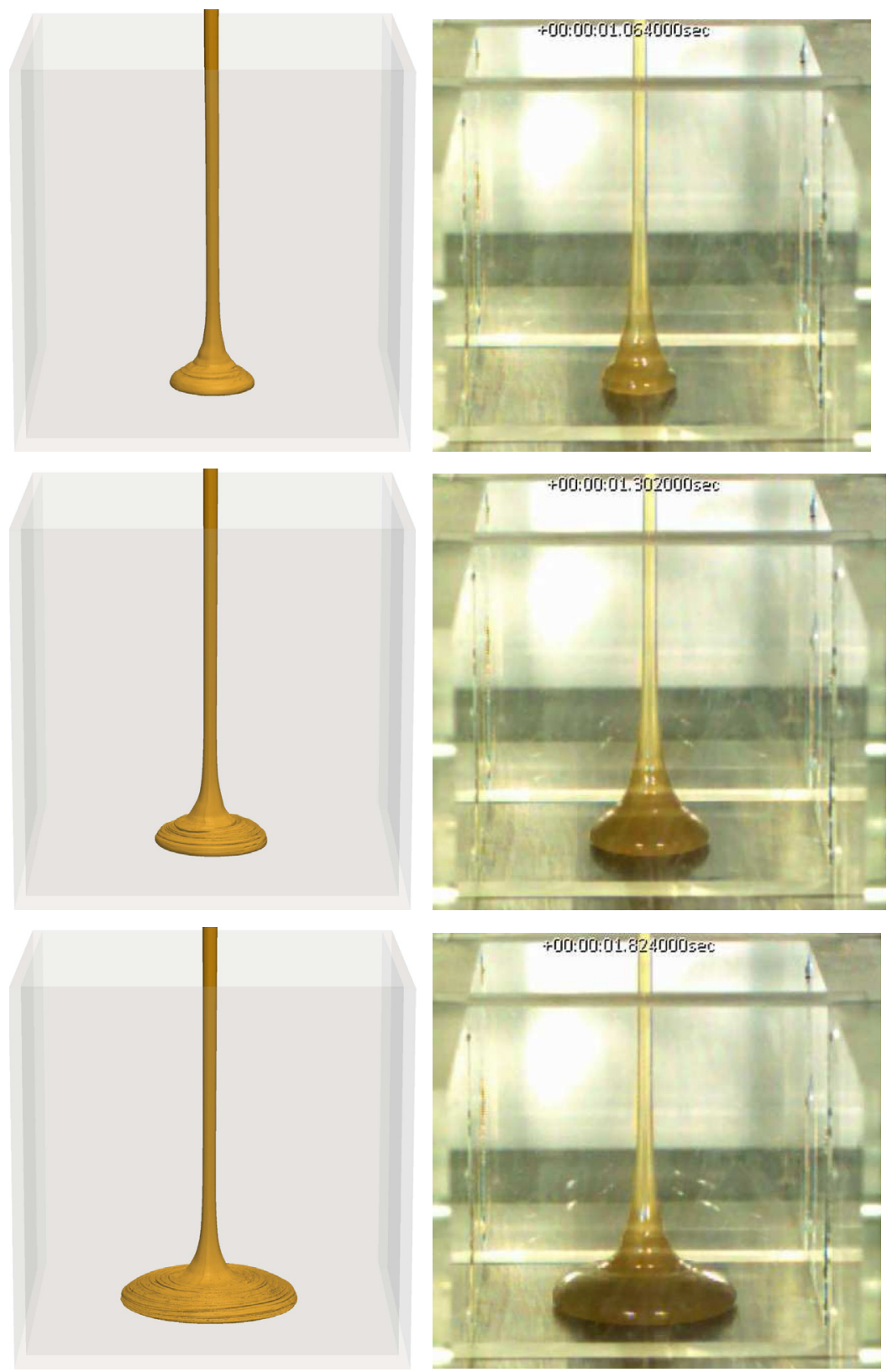

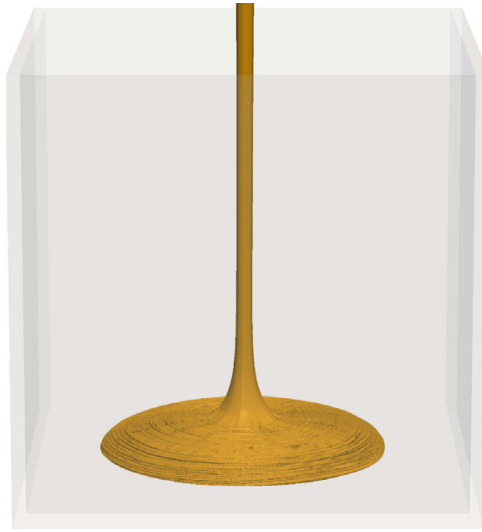

NUMERICAL

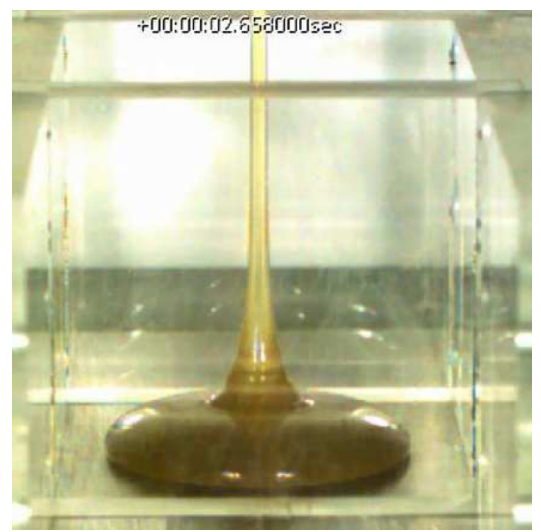

EXPERIMENTAL

Fig. 14. Snapshots from both numerical simulation and experimental observations of container filling for run $\mathbf{R 2}(\mathrm{Re}=0.97, \mathrm{Fr}=0.96)$. 

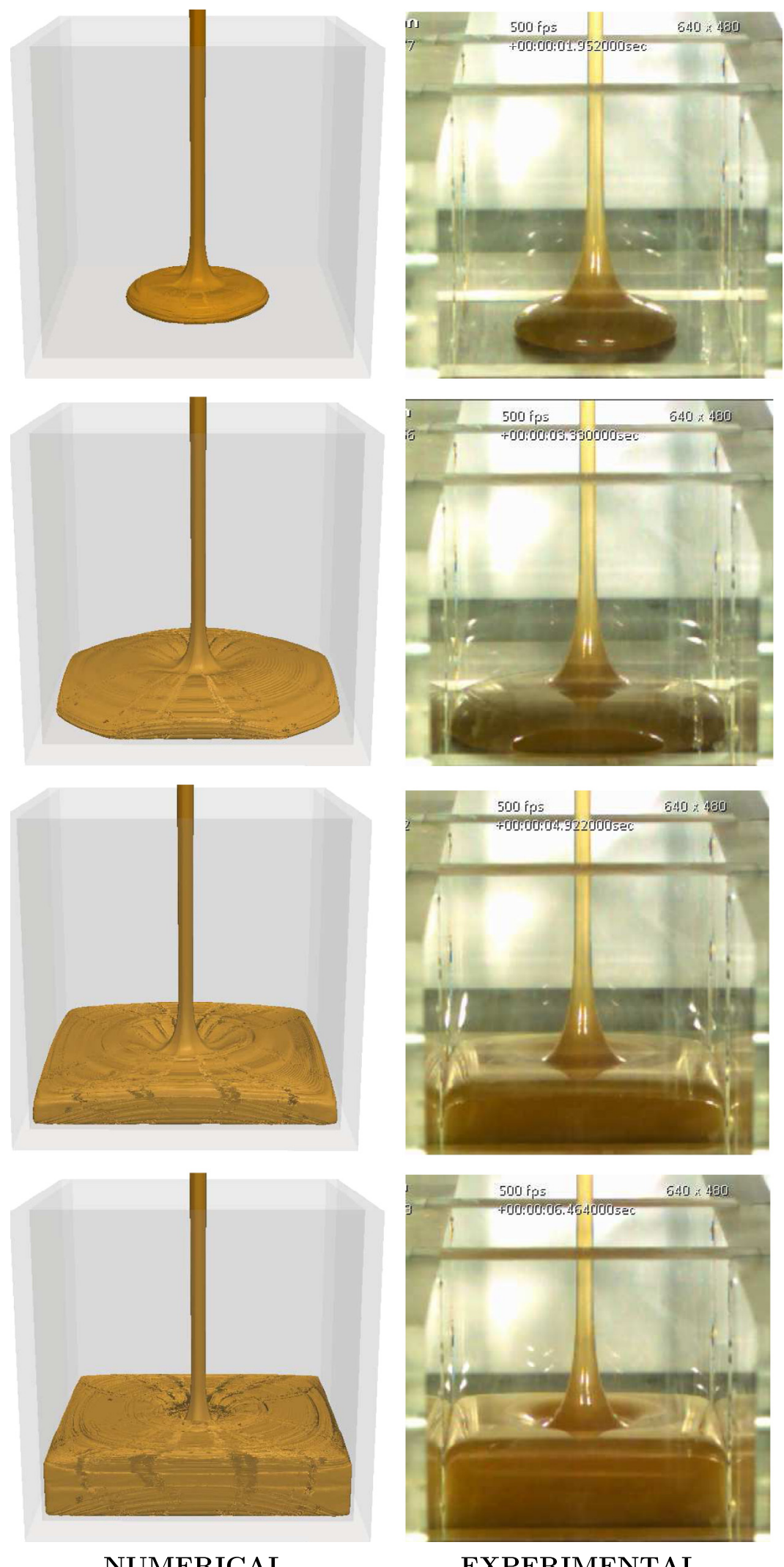

\section{EXPERIMENTAL}

Fig. 15. Snapshots from both numerical simulation and experimental observations of container filling for run $\mathbf{R 3}(\mathrm{Re}=1.93, \mathrm{Fr}=1.92)$. 

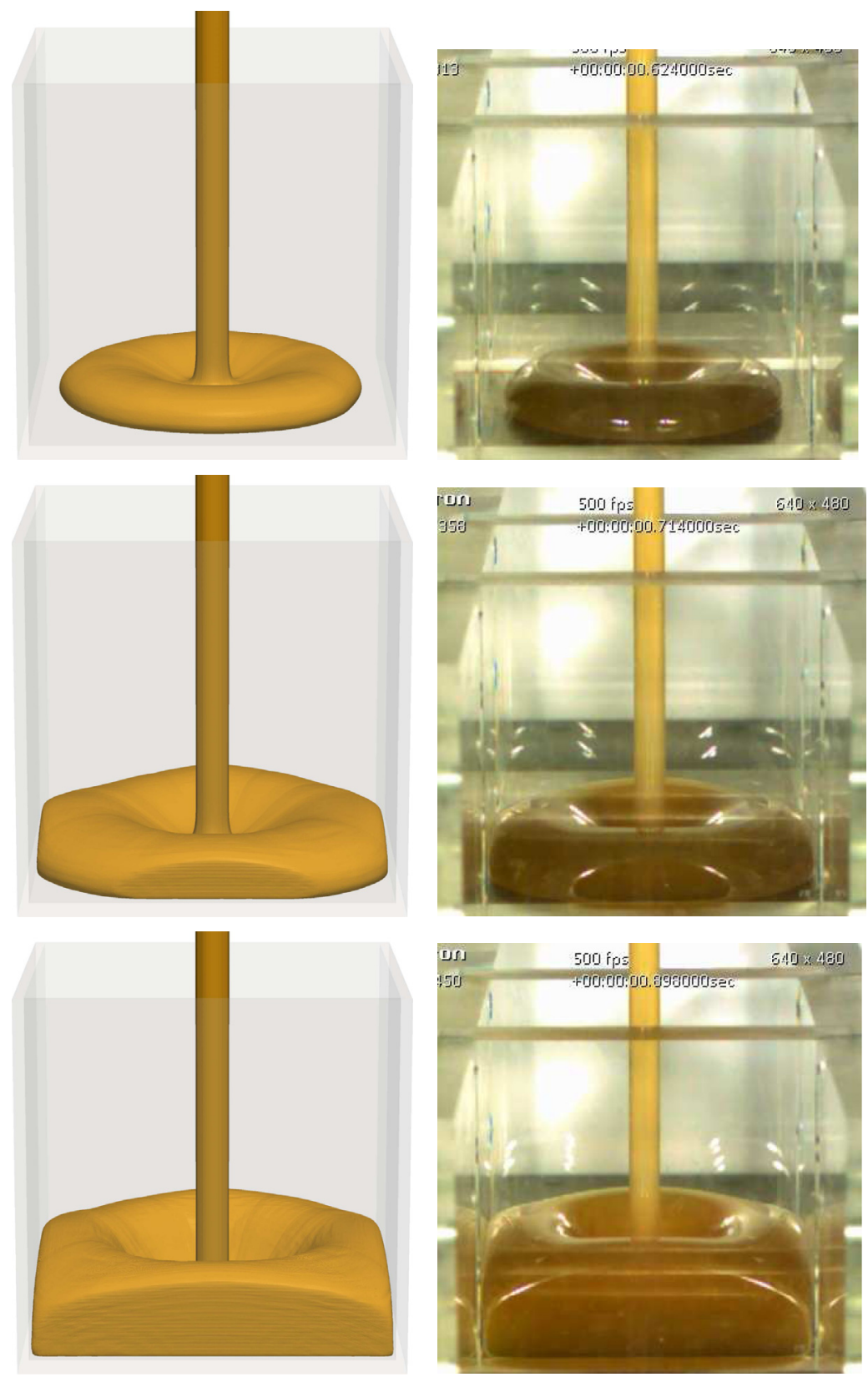

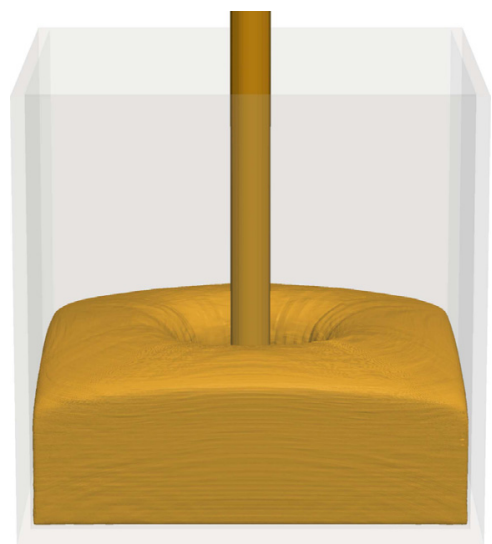

NUMERICAL

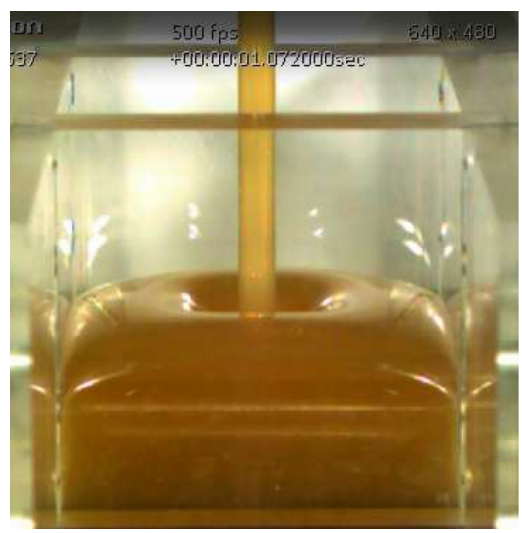

EXPERIMENTAL

Fig. 16. Snapshots from both numerical simulation and experimental observations of container filling for run $\mathbf{R 5}(\mathrm{Re}=24.16, \mathrm{Fr}=23.94)$ 


\section{Acknowledgements}

The Brazilian authors would like to acknowledge the financial support given by the funding agencies: $\mathrm{CNPq}$ - Conselho Nacional de Desenvolvimento Científico e Tecnológico (Grant Nos. 302631/ 2010-0, 301408/2009-2, 472514/2011-3), FAPESP - Fundação de Amparo a Pesquisa do Estado de São Paulo (Grant No. 2011/ 13930-0) and CAPES Grant Nos. BEX 2844/10-9 and 226/09 (CAPES-FCT). This work is part of the activities developed within the CEPID-CeMEAI FAPESP project Grant No. 2013/07375 - 0 and also benefits from the early collaboration within the framework of the University of São Paulo (Brazil) and University of Porto (Portugal) research agreements. The Portuguese authors gratefully acknowledge funding from Fundação para a Ciência e Tecnologia (FCT) under the project PEst-C/CTM/LA0025/2013 (Strategic Project - LA 25-2013-2014), project PTDC/MAT/121185/2010 and FED$\mathrm{ER}$, via FCT.

\section{References}

[1] Batchelor GK. An introduction to fluid dynamics. Cambridge University Press 1967.

[2] Castelo A, Tomé MF, Cesar CNL, McKee S. Freeflow3D: an integrated simulation system for three-dimensional free surface flows. Comput Vis Sci 2000;2:199-210.

[3] Cruickshank JO, Munson BR. Viscous-fluid buckling of plane axisymmetric jets. J Fluid Mech 1981;113:221-39.
4] B. Debbaut, R. Keunings, M.J. Crochet, POLYFLOW: a finite element package for simulating viscous and viscoelastic flows. In: Ladeveze P, Zienkiewicz OC, editors, Proceedings of European Conference on New Advances in Computational Structural Mechanics, Giens, France; 1991. p. 689-96.

[5] Ferreira VG, Tomé MF, Mangiavacchi N, Castelo A, Cuminato JA, Fortuna AO, et al. High-order upwinding and the hydraulic jump. Int J Num Meth Fluids 2002;39:549-83.

[6] Grande E, Laso M, Picasso M. Calculation of variable-topology free-surface flows using CONFESSIT. J Non-Newt Fluid Mech 2003;113:127-45.

[7] FLOW-3D SOFTWARE, Copyright 2000 - 2013 Flow Science, Inc.

[8] McKee S, Tomé MF, Ferreira VG, Cuminato JA, Castelo A, Sousa FS, et al. The MAC method. Comput. Fluids 2008;37:907-30.

[9] Oishi C, Tomé MF, Cuminato JA, McKee S. An implicit technique for solving 3D low Reynolds number moving free surface flows. J Comput Phys 2008;227:7446-68

[10] Ribe NM. Coiling of viscous jets. Proc Roy Soc Lon A - Math Phys Eng Sci 2004;460:3223-39.

[11] Roberts SA, Rao RR. Numerical simulations of mounding and submerging flows of shear-thinning jets impinging in a container. J Non-Newt Fluid Mech 2011;166:1100-15.

[12] Sousa FS, Mangiavacchi N, Castelo A, Tomé MF, Cuminato JA, McKee S. A fronttracking method for simulation of 3D multifluid flows with free surfaces. J Comput Phys 2004:198:469-99.

[13] Tomé MF, McKee S, Barratt L, Jarvis DA, Patrick AJ. An experimental and numerical investigation of container filling with viscous fluids. Int J Num Meth Fluids 1999:31:1333-53.

[14] Tomé MF, Castelo A, Cuminato JA, Mangiavacchi N, McKee S. GENSMAC3D: A numerical method for solving unsteady three-dimensional free surface flows. Int J Num Meth Fluids 2001;37:747-96.

[15] Tomé MF, McKee S. GENSMAC: a computational marker-and-cell method for free surface flows in general domains. J Comput Phys 1994;110:171-86. 